\title{
Thermodynamics and small quantum systems
}

\author{
Th.M. Nieuwenhuizen \\ Institute for Theoretical Physics, University of Amsterdam, \\ Valckenierstraat 65, 1018 XE Amsterdam, The Netherlands
}

November 11, 2018

\begin{abstract}
Small quantum systems non-weakly coupled to a bath become in the quantum regime surrounded by a cloud of photons or phonons, which modifies their thermodynamic behavior. Exactly solvable examples are the Brownian motion of a quantum particle in a harmonic confining potential and coupled to a harmonic quantum thermal bath, e.g. an ion in a Penning trap, and a spin immersed in a bosonic bath, as occurs in NMR physics. It appears that the Clausius inequality đ $Q \leq T \mathrm{~d} S$ can be violated. For non-adiabatic changes of system parameters the rate of energy dissipation can be negative, and, out of equilibrium, cyclic processes are possible which extract work from the bath. Experimental setups for testing some of the effects are discussed.
\end{abstract}

\section{Introduction}

The microscopic basis of thermodynamics is statistical physics and equilibrium is described by the Gibbs distribution. It is typically taken for granted that, when going to the quantum regime, the classical Gibbs distribution can just be replaced by its quantum analog. It is not often stressed that this is only allowed in case of weak coupling with the bath. When that coupling is not weak, the Gibbs state of the total system (subsystem+bath) leads, after tracing out the bath, to a non-Gibbsian state for the subsystem. This endangers (near-) equilibrium thermodynamics. We analyze the situation for two exactly solvable problems. 
A Letter on the thermodynamics of quantum Brownian motion appeared [1, and was discussed in the scientific literature [2] [3]. This encouraged many others to investigate foundations of the second law, see the proceedings of conference 'Quantum Limits to the Second Law', San Diego, July 2002 [4. Many details on the thermodynamics of the model were presented in ref. [5].

A thermodynamic analysis of the somewhat related spin-boson model appeared in ref. [6].

\section{Quantum Brownian motion}

The Hamiltonian for a harmonic oscillator in a bath of harmonic oscillators reads ("Caldeira-Leggett model") $\mathcal{H}=\mathcal{H}_{S}+\mathcal{H}_{B}+\mathcal{H}_{\mathrm{I}}$, with [5]

$$
\mathcal{H}=\frac{p^{2}}{2 m}+\frac{1}{2} a x^{2}+\sum_{i}\left[\frac{p_{i}^{2}}{2 m_{i}}+\frac{m_{i} \omega_{i}^{2} x_{i}^{2}}{2}\right]+\sum_{i}\left[-x_{i} x+\frac{c_{i}^{2}}{2 m_{i} \omega_{i}^{2}} x^{2}\right]
$$

The interaction Hamiltonian includes a self-interaction. In certain situations it is self-generated, else the prefactor of the total $x^{2}$-term should be large enough to make the splitting with a positive $a$ is possible. Examples of quantum Brownian motion are: fluctuation effects in Josephson junctions, low-temperature quantum transport, and quantum-optical systems (e.g. an ion in a Penning trap)

We assume for simplicity that the levels of the bath are equally spaced, $\omega_{i}=i \Delta$, and a very small level spacing $\Delta$ corresponds to a large, extensive bath, allowing a sharp definition of temperature, thus providing a basis for thermodynamics. The so-called spectral density $J(\omega)=\sum_{i} \frac{\pi c_{i}^{2}}{2 m_{i} \omega_{i}} \delta\left(\omega_{i}-\omega\right)$ is called Ohmic when $J(\omega)=\gamma \omega$ for small $\omega$; we shall consider the quasi-Ohmic Drude-Ullersma spectrum, where the large- $\omega$ behavior is cut-off at the Debye frequency $\Gamma$, i.e. $J(\omega)=\gamma \omega \Gamma^{2} /\left(\omega^{2}+\Gamma^{2}\right)$.

\section{a Stationary distribution.}

The Wigner function of the subsystem is long known. By considering a Gibbsian for the total system and summing out the bath one gets

$$
W(x, p)=\frac{1}{Z_{x}} \exp \left(-\frac{a x^{2}}{2 T_{x}}\right) \times \frac{1}{Z_{p}} \exp \left(-\frac{p^{2}}{2 m T_{p}}\right)
$$



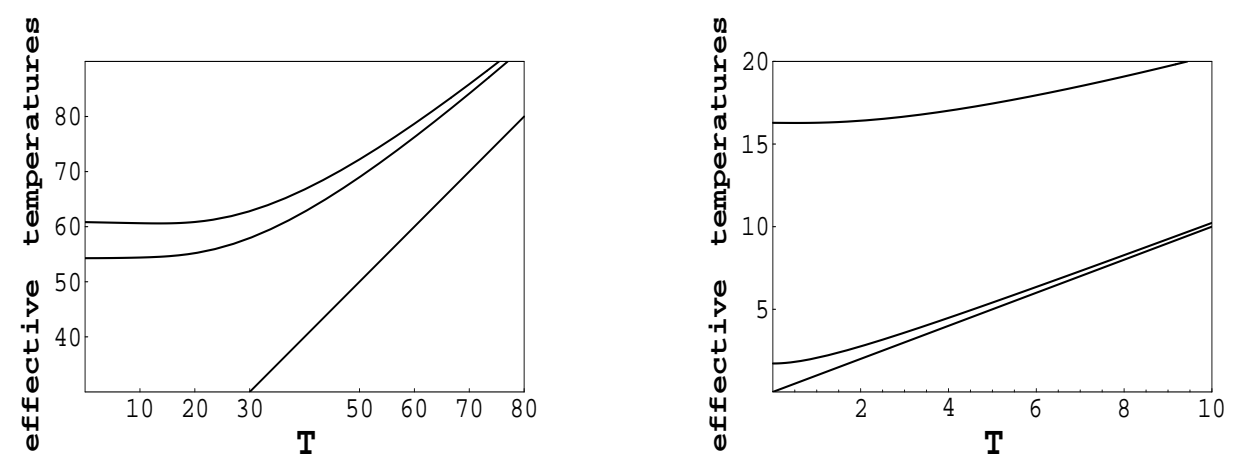

Figure 2.1: The effective temperatures $T_{p}$ (upper plot), $T_{x}$ (middle plot) versus the bath temperature $T$ (lower plot) for $\hbar \gamma /(4 \pi m)=1, \hbar \Gamma /(2 \pi)=$ 100. Left: $a m / \gamma^{2}=80$ (underdamping); Right: $a m / \gamma^{2}=0.2$ (moderate overdamping).

Here $T_{p}$ and $T_{x}$ are effective temperatures expressible in $\psi(z)=\Gamma^{\prime}(z) / \Gamma(z)$,

$$
\begin{aligned}
& T_{x}=T+\frac{\hbar a}{\pi m}\left\{\frac{\omega_{1}-\Gamma}{\left(\omega_{2}-\omega_{1}\right)\left(\omega_{3}-\omega_{1}\right)} \psi\left(\frac{\beta \hbar \omega_{1}}{2 \pi}\right)+\text { cyclic }\right\} \\
& T_{p}=T_{x}+\frac{\hbar \gamma \Gamma}{\pi m}\left\{\frac{\omega_{1}}{\left(\omega_{2}-\omega_{1}\right)\left(\omega_{3}-\omega_{1}\right)} \psi\left(\frac{\beta \hbar \omega_{1}}{2 \pi}\right)+\text { cyclic }\right\}
\end{aligned}
$$

where $\omega_{1,2,3}$ are the roots of $\omega^{3}-\Gamma \omega^{2}+(a+\gamma \Gamma) \omega / m-a \Gamma / m=0$ and where 'cyclic' refers to the two other terms generated by the cyclic interchange of the $\omega_{i}(i=1,2,3)$. In Figure 2.1 we present $T_{x}$ and $T_{p}$ as function of $T$ for a case of underdamping (left figure) and overdamping (right figure).

For a weakly coupled bath $(\gamma$ small $)$ one gets from this: $T_{p}=T_{x}=$ $\frac{1}{2} \hbar \omega_{0} \operatorname{coth}\left(\hbar \omega_{0} / 2 k T\right)$ with $\omega_{0}=\sqrt{a / m}$, the standard result obtained by assuming the Gibbs distribution for the particle. In the absence of a bath (i.e., first $\gamma \rightarrow 0$, then $T \rightarrow 0$ ) one would have $T_{p}=T_{x}=\frac{1}{2} \hbar \omega_{0}$.

When the coupling between subsystem bath (characterized by $\gamma$ ) is nonnegligible, one has $T_{p}>T_{x}>0$, even at $T=0$. The state (2.1) is nonGibbsian since $T_{p} \neq T_{x}$, which endangers (near-) equilibrium thermodynamics. 


\section{b Thermodynamic description for adiabatic changes}

The energy of the subsystem can be expressed through the Wigner function

$$
U=\left\langle\mathcal{H}_{\mathrm{S}}\right\rangle=\int \mathrm{d} p \mathrm{~d} x \mathcal{H}_{\mathrm{S}}(p, x) W(p, x) \equiv \int \mathrm{d} p \mathrm{~d} x \mathcal{H}_{\mathrm{S}} W
$$

where $\mathcal{H}_{\mathrm{S}}(p, x)=p^{2} / 2 m+a x^{2} / 2$. For small changes in the (effective) mass $m$ and/or the spring constant $a$, this allows to define the first law as

$$
\mathrm{d} U=\int \mathrm{d} p \mathrm{~d} x \mathcal{H}_{\mathrm{S}} \mathrm{d} W+\int \mathrm{d} p \mathrm{~d} x W \mathrm{~d} \mathcal{H}_{\mathrm{S}} \equiv \mathrm{d} Q+\mathrm{d} \mathcal{W}
$$

representing the heat and the work added to the subsystem, respectively. The latter is equal to the work extracted from the total system (subsystem+bath) 5 .

Violation of the Clausius inequality. The relation đQ $\leq T \mathrm{~d} S$ can now be tested at $T=0$, where $S$ is not needed. One finds for a change in $m$

$$
\mathrm{d} Q(T \rightarrow 0)=\left(\frac{\partial T_{p}}{\partial m}+\frac{\partial T_{x}}{\partial m}+\frac{T_{p}}{m}\right) \frac{\mathrm{d} m}{2}=\frac{\hbar \gamma}{2 \pi m^{2}} \mathrm{~d} m \neq 0 .
$$

For $\mathrm{d} m>0$ energy is supplied by the bath to the subsystem, even though $T=0$. It can be shown that this energy comes fully from the change in interaction energy of the cloud of bath modes around the subsystem [5].

A setup to test this violation in nanoscale electric circuits has been proposed; notice that the parameter dependence of $T_{x}$ was already confirmed [7].

Violation of the Landauer bound. For the erasure of one bit of information Landauer argued that an amount of heat $|₫ Q| \geq k T \ln 2$ should be dispersed. This is a special form of the Clausius inequality in the regime $\mathrm{d} Q<0$, $\mathrm{d} S=-\ln 2$. However, an explicit example for small $T$ shows that, in a process where work is added to the system, one can adsorb heat, $\mathrm{d} Q>0$, and nevertheless become more localized, $\mathrm{d} S<0[8$.

\section{c Non-adiabatic energy dispersion}

The work can be decomposed in the adiabatic part and the energy dispersion, $\mathrm{d} \mathcal{W}=\mathrm{đ} \mathcal{W}_{\text {adiabatic }}+\mathrm{đ} \Pi$. For a small, smooth and slow change of the spring constant, $a(t)=[1+\alpha(t)] a$, one gets at small $T$ to order $\alpha^{2}[5]$

$$
\frac{\mathrm{d} \Pi}{\mathrm{d} t}=T^{2} \dot{\alpha}^{2}+\dot{\alpha} \ddot{\alpha}-\dot{\alpha} \partial_{t}^{3} \alpha
$$




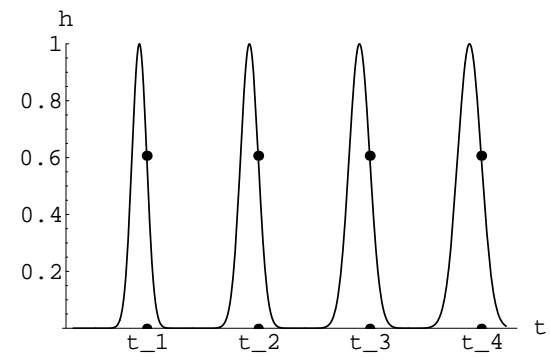

Figure 2.2: Schematic plot of the cyclic changes in the spring constant, where successive cycles are slower and slower. $h$ characterizes the size of the change and $t$ denotes the dimensionless time. The interval $-\infty<t<t_{1}$ marks the process that establishes the nonequilibrium state at $t=t_{1}$. The picture shows three full cycles, between the bullets.

where numerical factors have been left out. For a completed change the adiabatic term drops out and the dispersion brings $\Delta \mathcal{W}=\Delta \Pi=\int_{-\infty}^{\infty} \mathrm{d} t \mathrm{~d} \Pi / \mathrm{d} t=$ $\int_{-\infty}^{\infty} \mathrm{d} t\left(T^{2} \dot{\alpha}^{2}+\ddot{\alpha}^{2}\right)>0$, confirming the Thomson formulation of the second law (cycles cost work) 9 .

When $T$ is small and $\alpha$ is a slow function of time, the $\dot{\alpha} \ddot{\alpha}$ term dominates; only in its full integral its positive and negative parts cancel. Thus a negative $\frac{d \Pi}{\mathrm{d} t}$ is possible. This poses a counterexample to the formulation of the second law stating that the rate of energy dispersion be positive [10].

The fact that at low $T$ the $\dot{\alpha} \ddot{\alpha}$-term is leading can be exploited. Cycles in $a(t)$ can be constructed in which energy is extracted. In figure 2.2 we show bell-shaped curves for $\alpha(t)=\alpha_{\max } h(t)$, which become slower and slower. That construction is needed to let the $\dot{\alpha} \ddot{\alpha}$-term overcome the inherent loss due to the first and third term [5].

The total dispersion is positive, because more work is needed to create the non-equilibrium state at $t_{1}$ than can be recovered in the cycles. Nevertheless this can be called a "Perpetuum mobile of second kind" [5]. The maximal number of work extraction cycles is of order $1 / T$. Optimal extraction occurs, however, by making one cycle, due to the trend for dispersion in completed cycles. 


\section{d Entropic considerations}

Entropy is historically a newer concept than heat and work. It has come as a surprise that in the present field entropy arguments fail even quicker. With the Clausius inequality being broken, there is no sense in defining a "thermodynamic" entropy $S_{\text {thermo }}=\int_{0}^{T} \mathrm{~d} T^{\prime}\left(\mathrm{d} Q / \mathrm{d} T^{\prime}\right) / T^{\prime}$; this quantity would not have a statistical interpretation, and, actually, the integral would not even converge at its lower bound.

The most common formulation of the second law is: the (coarse grained) entropy of a closed system cannot decrease. It becomes for an open subsystem (immersed in its bath): the rate of entropy production cannot be negative. By defining the flow of Boltzmann entropy, we came to the conclusion that the rate of Boltzmann entropy production can be negative already for moderate temperatures [5].

\section{Bath generated work extraction in two- level systems}

Another application is the spin-boson model, often used in NMR and ESR physics, quantum optics and spintronics. Its Hamiltonian reads [6]

$$
\begin{aligned}
\mathcal{H} & =\mathcal{H}(\Delta)=\mathcal{H}_{S}+\mathcal{H}_{B}+\mathcal{H}_{I}, \\
\mathcal{H}_{S} & =\frac{\varepsilon}{2} \hat{\sigma}_{z}+\frac{\Delta(t)}{2} \hat{\sigma}_{x}, \quad \mathcal{H}_{B}=\sum_{k} \hbar \omega_{k} \hat{a}_{k}^{\dagger} \hat{a}_{k}, \quad \mathcal{H}_{I}=\frac{1}{2} \sum_{k} g_{k}\left(\hat{a}_{k}^{\dagger}+\hat{a}_{k}\right) \hat{\sigma}_{z} .
\end{aligned}
$$

This is a spin- $\frac{1}{2}$ interacting with a bath of harmonic oscillators. $\hat{\sigma}_{x}, \hat{\sigma}_{y}$ and $\hat{\sigma}_{z}=-i \hat{\sigma}_{x} \hat{\sigma}_{y}$ are Pauli matrices, and $\hat{a}_{k}^{\dagger}$ and $\hat{a}_{k}$ are the creation and annihilation operators of the bath oscillator with the index $k$, while the $g_{k}$ are the coupling constants. For an electron in a magnetic field $B, \varepsilon=\bar{g} \mu_{B} B$ is the energy, with $\bar{g}$ the gyro-magnetic factor and $\mu_{B}$ the Bohr magneton. We take the transversal field $\Delta(t)=0$ except during pulses. This model is a prototype of a variety of physical systems [11, and known to be exactly solvable [11, 12, since the $z$-component of the spin is conserved, and with it the spin energy. Physically it means that we restrict ourselves to times much less than $\mathcal{T}_{1}$ (relaxation time of $\hat{\sigma}_{z}$ ). In ESR physics 13 , the model represents an electron-spin interacting with a bath of phonons, for NMR it can represent a nuclear spin interacting with a spin bath, since in certain natural limits the 
latter can be mapped to the oscillator bath. In quantum optics it is suitable for describing a two-level atom interacting with a photonic bath [14.

One typically takes a quasi-Ohmic spectral density [1] $J(\omega)=\sum_{k} g_{k}^{2} \delta\left(\omega_{k}-\right.$ $\omega) /\left(\hbar \omega_{k}\right)=g \hbar \exp (-\omega / \Gamma) / \pi$, where $g$ is a dimensionless damping constant and the exponential cuts off the coupling at $\omega \gg \Gamma$, the maximal frequency of the bath. Since $\Delta=0$, one has conservation of $\hat{\sigma}_{z}(t)=\hat{\sigma}_{z}(0)$

Separated initial state. To describe situations, where the spin was suddenly brought into the contact with the bath, e.g. an electron injected into semiconductor, atom injected into a cavity, or exciton created by external radiation, we make the assumption that initially, at $t=0$, the spin and the bath are in a separated state, the latter being Gibbsian, $\rho(0)=$ $\rho_{S}(0) \otimes \exp \left(-\beta \mathcal{H}_{B}\right) / Z_{B}$, where $\rho_{S}(0)$ is the initial density matrix of the spin. In terms of the Larmor frequency $\omega_{0}=\varepsilon / \hbar$ this brings for the evolution of the transverse components

$$
\begin{aligned}
& \left\langle\hat{\sigma}_{x}(t)\right\rangle=\left[\cos \omega t\left\langle\hat{\sigma}_{x}(0)\right\rangle-\sin \omega t\left\langle\hat{\sigma}_{y}(0)\right\rangle\right] e^{-t / \mathcal{T}_{2}}, \\
& \left\langle\hat{\sigma}_{y}(t)\right\rangle=\left[\cos \omega t\left\langle\hat{\sigma}_{y}(0)\right\rangle+\sin \omega t\left\langle\hat{\sigma}_{x}(0)\right\rangle\right] e^{-t / \mathcal{T}_{2}},
\end{aligned}
$$

where $\mathcal{T}_{2}=\hbar /(g T)$ is the transversal decay time.

The von Neumann entropy equals $S=-\operatorname{tr} \rho_{S} \ln \rho_{S}=-p_{1} \ln p_{1}-p_{2} \ln p_{2}$, where $p_{1,2}=\frac{1}{2} \pm \frac{1}{2}|\langle\vec{\sigma}\rangle|$. The decay of $\left\langle\sigma_{x, y}(t)\right\rangle$ makes the von Neumann entropy increase. Since there is no heat flow - the energy is conserved - this is in agreement with a formulation of the second law: the entropy of a closed system, or of an open system without energy transfer, cannot decrease.

A sudden pulse. A fast rotation around the $x$-axis is described by taking $\Delta \neq 0$ during a short time [13], yielding $\left(\hat{\sigma}_{y, z}\right)^{\prime}=\hat{\sigma}_{y, z} \cos \theta \pm \hat{\sigma}_{z, y} \sin \theta$. During the sudden switchings of $\Delta(t)$ the density matrix is just rotated.

Our main interest is work extraction from the bath. In order to ensure that the pulse does not change the energy of the spin, we first consider the case $\varepsilon=0$, where the spin has no energy. For small $g, \theta=-\pi / 2$ and $t \gg 1 / \Gamma$ the work appears to be

$$
W_{1}=\frac{g \hbar \Gamma}{2 \pi}+\frac{g T}{2}\left\langle\hat{\sigma}_{x}(0)\right\rangle e^{-t / \mathcal{T}_{2}}
$$

If for a fixed $t$, temperature is neither too large nor too small, $T e^{-t / \mathcal{T}_{2}}>$ $\hbar \Gamma / \pi$, work can be extracted $\left(W_{1}<0\right)$, provided the spin started in a coherent state $\left\langle\hat{\sigma}_{x}(0)\right\rangle=-1$. This possibility to extract work from the equilibrated bath $(t \gg 1 / \Gamma)$ contradicts the Thomson's formulation of the second law out 
of equilibrium. It disappears on the timescale $\mathcal{T}_{2}$, because then the spin looses its coherence, $\left\langle\hat{\sigma}_{x, y}(t)\right\rangle \rightarrow 0$. Classical variations $( \pm \pi$ pulses, which do not involve coherence) can extract work only from a non-thermalized bath, i.e. for times $\sim 1 / \Gamma$. Thus, the effect is essentially quantum mechanical.

Initial preparation via a rotation. Starting from a Gibbsian of the total system, at $t=0$ the spin is rotated ("zeroth pulse") over an angle $-\frac{1}{2} \pi$ around the $y$-axis. This maps $\hat{\sigma}_{x} \rightarrow \hat{\sigma}_{z}, \hat{\sigma}_{z} \rightarrow-\hat{\sigma}_{x}$. Such a state models the optical excitation of the spin, as done in NMR and spintronics. The problem remains exactly solvable. Taking $\theta=-\frac{1}{2} \pi$ in the first pulse one now gets

$$
W_{1} \approx \frac{g \hbar \Gamma}{2 \pi}-\left[\frac{\varepsilon}{2} \sin \omega_{0} t+\frac{g T}{2} \cos \omega_{0} t\right] \tanh \frac{\beta \varepsilon}{2} e^{-t / \mathcal{T}_{2}}
$$

The work decomposes as $W_{1}=\Delta U-\Delta Q$, with

$$
\Delta Q \approx \frac{g}{2}\left[-\frac{\hbar \Gamma}{\pi}+T \cos \omega_{0} t \tanh \frac{\beta \varepsilon}{2} e^{-t / \mathcal{T}_{2}}\right],
$$

quite similar to $-W_{1}$ of Eq. (3.10). An interesting case is where work is performed by the total system $\left(W_{1}<0\right)$ solely due to heat from the bath $(\Delta Q>0, \Delta U=0)$. This process, possible by choosing $t \approx 2 \pi n / \omega_{0}$ with integer $n$, involves a $\Delta Q>0, \Delta S=0$, which violates the Clausius inequality.

The work needed at $t=0$ to rotate the spin is $W_{0}=(\varepsilon / 2) \tanh [\beta \varepsilon / 2]+$ $g \hbar \Gamma /(2 \pi)$. The extracted work is smaller, confirming Thomson's equilibrium formulation for cyclic changes ( $\Delta=0$ before and after the pulses) [9].

Two pulses in a rotated initial Gibbsian state. If there are many (very weakly interacting) spins, each in a slightly different external field, an inhomogeneous broadening of the $\omega_{0}=\varepsilon / \hbar$ line occurs, for which we assume the distribution

$$
p\left(\omega_{0}\right)=\frac{2}{\pi} \frac{\left[\mathcal{T}_{2}^{*}\right]^{-1}}{\left(\omega_{0}-\bar{\omega}_{0}\right)^{2}+\left[\mathcal{T}_{2}^{*}\right]^{-2}}
$$

with average $\bar{\omega}_{0}$ and inverse width $\mathcal{T}_{2}^{*}$, typically much smaller than $\mathcal{T}_{2}$. In this case the gain for a single pulse is washed out, leaving only the loss $\Delta Q=-g \hbar \Gamma / 2 \pi$, so two pulses are needed. We consider again the rotated initial Gibbsian state, and perform a first $-\frac{1}{2} \pi$ pulse around the $x$-axis at time $t_{1}$ and a second $\frac{1}{2} \pi$ pulse at time $t_{2}$ (the standard $\frac{1}{2} \pi, \pi$ combination would not expose an interesting role of the bath). For the total work $W=W_{1}+W_{2}$ the averaging over $\omega_{0}$ brings

$$
W=\frac{g \hbar \Gamma}{\pi}-\frac{\hbar}{4} e^{-t_{2} / \mathcal{T}_{2}} e^{-|\Delta t| / \mathcal{T}_{2}^{*}} \tanh \frac{\beta \hbar \bar{\omega}_{0}}{2} \times
$$




$$
\left\{\bar{\omega}_{0} \sin \bar{\omega}_{0} \Delta t+\left[\frac{1}{\mathcal{T}_{2}}-\frac{\operatorname{sg}(\Delta t)}{\mathcal{T}_{2}^{*}}\left(1+\frac{\beta \hbar \bar{\omega}_{0}}{\sinh \beta \hbar \bar{\omega}_{0}}\right)\right] \cos \bar{\omega}_{0} \Delta t\right\}
$$

For $\Delta t \equiv t_{2}-2 t_{1}$ near $2 \pi n / \bar{\omega}_{0}$ such that the odd terms cancel, this again exhibits work extracted solely from the bath.

Feasibility. Let us notice that work and heat were measured in NMR experiments more than 35 years ago [15] and this technique (glue the sample on a copper wire and measure the change in its resistance) can be used to test the violation of the Clausius inequality. The ongoing activity for implementation of quantum computers provides experimentally realized examples of two-level systems, which have sufficiently long $\mathcal{T}_{2}$ times, and admit external variations on times smaller than $\mathcal{T}_{2}$ : $(i)$ for atoms in optical traps $\mathcal{T}_{2} \sim 1 \mathrm{~s}$, $1 / \Gamma \sim 10^{-8} \mathrm{~s}$, and there are efficient methods for creating non-equilibrium initial states and manipulating atoms by external laser pulses [16]; (ii) for an electronic spin injected or optically excited in a semiconductor $\mathcal{T}_{2} \sim 1 \mu \mathrm{s}$ [17]; (iii) for an exciton created in a quantum dot $\mathcal{T}_{2} \sim 10^{-9} \mathrm{~s}$ [18] (in cases (ii) and (iii) $1 / \Gamma \sim 10^{-13} \mathrm{~s}$ and femtosecond $\left(10^{-15} \mathrm{~s}\right)$ laser pulses are available); ( $i v)$ in NMR physics $\mathcal{T}_{2} \sim 10^{-6}-1$ s and the duration of pulses can be comparable with $1 / \Gamma \sim 1 \mu \mathrm{s}$.

\section{e Bath-induced gain without inversion}

A two-level system with population inversion, i.e. with a negative temperature, is a working mechanism of lasers and masers. In this context a bath is typically considered as a source of undesirable noises and relaxation towards equilibrium [14]. The bath can nevertheless play a totally different role, namely in assisting work extraction (gain) by means of a positive temperature state in the two-level system. In absence of coupling to the bath such an effect is strictly prohibited by the second law applied to a positive temperature spin state [9].

We consider separated initial conditions with $\left\langle\hat{\sigma}_{x}(t)\right\rangle=\left\langle\hat{\sigma}_{y}(t)\right\rangle=0$, and apply a $-\frac{1}{2} \pi$ pulse around the $x$-axis at time $t_{0}=0^{+}$, and a $\frac{1}{2} \pi$ pulse at $t$. For $t \gg 1 / \Gamma$ the work $W=\Delta U-\Delta Q$ is set by:

$$
\begin{aligned}
& \Delta U=-\frac{\varepsilon}{2}\left[1-e^{-t / \mathcal{T}_{2}} \cos \omega_{0} t\right]\left\langle\hat{\sigma}_{z}\right\rangle+\frac{g \varepsilon}{4} e^{-t / \mathcal{T}_{2}} \sin \omega_{0} t \\
& \Delta Q=-\frac{g \hbar \Gamma}{\pi}+\frac{1}{2} g T e^{-t / \mathcal{T}_{2}} \sin \omega_{0} t\left\langle\hat{\sigma}_{z}\right\rangle
\end{aligned}
$$

In the inversion-free case the initial state of the spin is a Gibbsian connected with temperature $T_{0}=1 / \beta_{0}$, for which $\left\langle\hat{\sigma}_{z}\right\rangle=-\tanh \frac{1}{2} \beta_{0} \varepsilon \leq 0$. For $T_{0}=\infty$ 
one has the completely random state, $\left\langle\hat{\sigma}_{x, y, z}\right\rangle=0$. The work $W$ can be negative (gain) provided $\varepsilon>4 \hbar \Gamma / \pi$. This situation can be met in quantum optical two-level systems [14, 16] and in NMR [19]. This mechanism concerns work extraction with help of the bath (it disappears for $g \rightarrow 0$ ), but not from the bath, since now $\Delta Q<0$. The origin of the effect is that although the state of the spin was completely disordered initially, the first pulse does generate some coherence. Due to back-reaction of the bath one has after the pulses $\left\langle\hat{\sigma}_{y}(t)\right\rangle=\frac{1}{2} g \exp \left(-t / \mathcal{T}_{2}\right) \sin \omega_{0} t$

At finite $T_{0}$ the term $\Delta U$ can still be negative when $T_{0}>\varepsilon / g$, which can be met for not-too-small $g$, a condition anyhow needed for having a sizeable effect. From a thermodynamic point of view the gain can just be seen as a flow of energy from a high temperature (of the spin) to a lower one (of the bath), and the outside world (gain).

There exist other mechanisms for inversionless gain [14; the crucial difference is that they operate with (at least) three-level systems (atoms), and - most importantly - the effect appears due to special, non-thermal states of the atom itself. Frequently these states involve a hidden inversion [14.

\section{Exact theorems}

There are a few exact theorems that help to reach a final formulation of the second law. They all start from initial Gibbsian equilibrium.

1 The Thomson formulation (cycles cost work) allows an exact proof when starting in equilibrium [9].

2 The amount of heat that can go from an equilibrium low temperature bath to an equilibrium high temperature bath is limited and proportional to the interaction energy. In particular, this forbids steady energy currents from low to high $T$ [20]

In previously discussed models these theorems can be checked whenever they apply, that is to say, when the initial state is Gibbsian. 


\section{What went wrong with standard thermo- dynamics?}

The present research considers small quantum systems not so weakly coupled to their baths, that the interaction energy can be neglected.

For such systems a number of complications show up in the formulation of thermodynamics. We have indeed shown counterexamples against various formulations, in particular those involving the entropy. Measurements have been proposed to test the most obvious one, the breakdown of the Clausius inequality, e.g. for pulses in NMR-physics. This immediately implies the possibility to violate the Landauer bound for erasure of information. Further unexpected aspects are a negative rate of energy dispersion and the possibility of work extraction cycles.

On the other hand, there are exact theorems which save some formulations (mostly involving only energy or work) provided the initial state is Gibbsian and the work source is not correlated to the test system and its bath.

The aim of the present field is to establish thermodynamically unexpected energy flows on microscopic or mesocopic scales. The field of laser physics is a promising play ground for such phenomena.

\section{References}

[1] A.E. Allahverdyan and Th.M. Nieuwenhuizen, Extraction of work from a single thermal bath in the quantum regime, Phys. Rev. Lett. 85 (2000) 1799

[2] Philip F. Schewe and Ben Stein, New frontiers of thermodynamics, http://www.aip.org/enews/physnews/2000/split/pnu494-1.htm

[3] Peter Weiss, Breaking the law: Can quantum mechanics + thermodynamics = perpetual motion ?, Sci. News (Washington D.C.) 158, 234 (2000)

[4] Quantum Limits to the Second Law, D. Sheehan, ed., AIP Conference Proceedings 643 (2002) 
[5] Th.M. Nieuwenhuizen and A.E. Allahverdyan, Statistical thermodynamics of quantum Brownian motion: Construction of perpetuum mobile of the second kind, Phys. Rev. E 66, 036102 (2002)

[6] A.E. Allahverdyan and Th.M. Nieuwenhuizen, Bath-generated work extraction and inversion-free gain in two-level systems, J. Phys. A: Math. Gen. 36, 875 (2003)

[7] A.E. Allahverdyan and Th.M. Nieuwenhuizen, On testing the violation of the Clausius inequality in nanoscale electric circuits, Phys. Rev. B 66, 115309 (2002); also: Virtual J. Nanoscale Sci. \& Techn., 6, Issue 13 (2002)

[8] A.E. Allahverdyan and Th.M. Nieuwenhuizen, Breakdown of the Landauer bound for information erasure in the quantum regime, Phys. Rev. E 64, 056117 (2001)

[9] A.E. Allahverdyan and Th.M. Nieuwenhuizen, A mathematical theorem as the basis for the second law: Thomson's formulation applied to equilibrium, Physica A 305, 542 (2002).

[10] P. Kondopudi and I. Prigogine, Modern thermodynamics: from heat engines to dissipative structures, (Wiley, West Sussex, UK, 1998).

[11] A. J. Leggett, S. Chakravarty, A. T. Dorsey, M. P. A. Fisher, A. Garg and W. Zwerger, Rev. Mod. Phys., 59, 1 (1987).

[12] J. Luczka, Physica A, 167, 919 (1990).

[13] A. Abragam, Principles of Nuclear Magnetism, (Clarendon, Oxford, 1961); R.R. Ernst, G. Bodenhausen, A. Wokaun, Principles of Nuclear Magnetic Resonance in One and Two Dimensions, (Clarendon, Oxford, 1987).

[14] M. Scully and S. Zubairy, Quantum Optics, (Cambridge University Press, 1997). O. Kocharovskaya, Phys. Rep., 219, 175, (1992).

[15] J. Schmidt and I. Solomon, J. Appl. Phys. 37, 3719 (1966).

[16] J.I. Cirac and P. Zoller, Phys. Rev. Lett. 74, 4091 (1995); D. Frese, B. Ueberholz, S. Kuhr, W. Alt, D. Schrader, V. Gomer, and D. Meschede, Phys. Rev. Lett. 85, 3777 (2000). 
[17] J.M. Kikkawa and D.D. Awschalom, Science, 287, 473 (2000).

[18] N. H. Bonadeo, G. Chen, D. Gammon, D.S. Katzer, D. Park, and D.G. Steel, Science, 282, 1473 (1998); Nonlinear Nano-Optics: Probing One Exciton at a Time, Phys. Rev. Lett. 81, 2759 (1998).

[19] S. Dattagupta, G. K. Shenoy, B. D. Dunlap, and L. Asch, Phys. Rev. B 16, 3893 (1977).

[20] A. E. Allahverdyan, R. Balian and Th.M. Nieuwenhuizen, Thomson's formulation of the second law: an exact theorem and limits of its validity, Ref [4] pp. 35-40; cond-mat/0208563 